\title{
Optical properties of 2D photonic structures fabricated by direct laser writing
}

\author{
Artem Sinelnik $^{1} \cdot$ Kirill Samusev $^{1,2} \cdot$ Mikhail Rybin $^{1,2} \cdot$ Mikhail Limonov $^{1,2}$
}

(C) Springer Nature Switzerland AG 2019

\begin{abstract}
This paper presents the results of fabrication and investigation of different $2 \mathrm{D}$ photonic structures with the square $\mathrm{C}_{4 v^{\prime}}$ hexagonal $\mathrm{C}_{6 \mathrm{v}}$ and pentagonal local $\mathrm{C}_{5 \mathrm{v}}$ symmetry. The samples were created using the direct laser writing technique. The number of submicron scatterers varied from 25 to 10,000, and the lattice parameters varied in different samples in the range of $0.5 \mu \mathrm{m} \leq a \leq 8.0 \mu \mathrm{m}$. Our experimental and theoretical studies demonstrate that 2D photonic structures reveal many remarkable optical effects. Created ordered and disordered photonic structures demonstrate a great variety of optical phenomena observed on a far-field screen placed behind the sample. When the random fluctuations of the orientation for individual rods were introduced to the perfect fishnet photonic structure, a crossover from Laue diffraction to speckle patterns was observed and investigated.
\end{abstract}

Keywords Light scattering · Laue diffraction · SEM images · Photonic structure $\cdot$ Metasurface $\cdot$ Direct laser writing

\section{Introduction}

During the interaction of light with photonic structures, diffraction, refraction, reflection, and absorption result in light scattering patterns. Our goal is to provide a comprehensive description of optical diffraction spectra of 2D dielectric photonic structures possessing low dielectric contrast. Using the direct laser writing technique [1-3], we fabricate a variety of $2 \mathrm{D}$ photonic microstructures with different symmetry, lattice constant, number of elements, and degree of disorder. Unambiguous interpretation of the effects observed in optical spectra can be obtained only on the basis of extended structural information using scanning electron microscopy (SEM). To evaluate the complex response function of $2 \mathrm{D}$ photonic structures, we check several models and compare theoretical and experimental results.

In our previous works [4, 5], we studied a fine structure of the diffraction patterns that is clearly observed for samples with rather a small number of scatterers of $2 \mathrm{D}$ structures with the orthogonal $C_{2 v}$, square $C_{4 v}$ and hexagonal $\mathrm{C}_{6 \mathrm{v}}$ symmetry. We demonstrated that one can define the number of scatterers $N$ directly from the experimental optical diffraction patterns. For conventional 2D photonic films with a large number of scatterers, all maxima merge into spectrum and cannot be resolved in the averaged profile of the diffraction patterns. The transformation of diffraction patterns from a 2D single layer toward a bulk $3 \mathrm{D}$ structure was demonstrated by the example of optical studies of synthetic opals $a-\mathrm{SiO}_{2}$ [6].

Here, we study experimentally optical diffraction from fabricated ordered and disordered 2D woodpile structures as well as from the Penrose tilings. When the random fluctuations of the orientation for individual rods were introduced to the ideal woodpile photonic structure, a crossover from Laue diffraction to speckle patterns was observed and investigated experimentally and numerically. Laser speckle [7] is an interference pattern produced by light scattered from different parts of the disordered structure. The scattering intensity at any point on the screen is determined

Mikhail Limonov, M.Limonov@mail.ioffe.ru| ${ }^{1}$ ITMO University, St. Petersburg, Russia $197101 .{ }^{2}$ loffe Institute, St. Petersburg, Russia 194021.

SN Applied Sciences (2019) 1:1213 | https://doi.org/10.1007/s42452-019-1220-y 
by the algebraic addition of all the wave amplitudes at this point.

\section{Experimental section}

\subsection{Samples preparation and characterization}

In this study, to fabricate different 2D photonic microstructures, we employ the two-photon polymerization method, which is also called direct laser writing (DLW) [1-3]. The method is based on the nonlinear two-photon polymerization of a photosensitive material (negative or positive) in the focus of a laser beam. A high resolution of the DLW method is due to the intensity-threshold character of the polymerization process which occurs in a region with sizes significantly smaller than the size of the focused beam. This technique makes it possible to form a 3D photonic crystal with a transverse resolution below $100 \mathrm{~nm}$.

To realize the direct laser writing technique, we use the installation and software from Laser Zentrum Hannover (Germany). The structures were fabricated using a hybrid organic-inorganic material based on zirconium propoxide with an Irgacure 369 photo-initiator (Ciba Specialty Chemicals Inc., Basel, Switzerland). The advantage of this material is low shrinkage upon polymerization, which guarantees a perfect correspondence of the resulting structure to a suggested mathematical model. The polymerization was performed with a train of femtosecond pulses (wavelength was $780 \mathrm{~nm})$ at a repetition frequency of $80 \mathrm{MHz}(12.5 \mathrm{~ns}$ between consecutive pulses) from a $50 \mathrm{fs}$ TiF-100F laser (Avesta-Project, Russia). Laser radiation was focused in the photoresist volume through the glass substrate with a $100 \times$ oil-immersion microscope objective with numerical aperture $N A=1.4$. The structures were fabricated by means of raster scanning in a layer-by-layer format. The scanning path of the laser focus is moved along a 2D scanning path in the $x y$-plane, while translation of the beam spot in the $z$-axis enables processing of 3D photonic structures.

The structures of fabricated samples are shown in Fig. 1. As an example, we describe in more detail the structure of the ordered and disordered fishnet 2D crystals. A woodpile structure [8] consisting of only two layers is called a fishnet structure. With the square dielectric rod as building block, two types of fishnet structures were created: a perfectly ordered arrangement structure (Fig. 1b) and a random arrangement of rods for glassy metasurfaces (two $x y$-layers) Fig. 1c. We fabricated the disordered fishnet structures as follows. Each individual rod in the $x y$-layer was rotated around its center (along the $x$ - or $y$-axis) by random angle $\alpha_{i}$ with respect to the ordered structure. We used two different kinds of random fluctuations, namely distribution functions: $\sigma=p \frac{\pi}{4}, 0 \leq p \leq 1$ is the dispersion of $\alpha$ and uniform distribution $-\alpha_{\max } \leq \alpha_{i} \leq \alpha_{\max }$ with
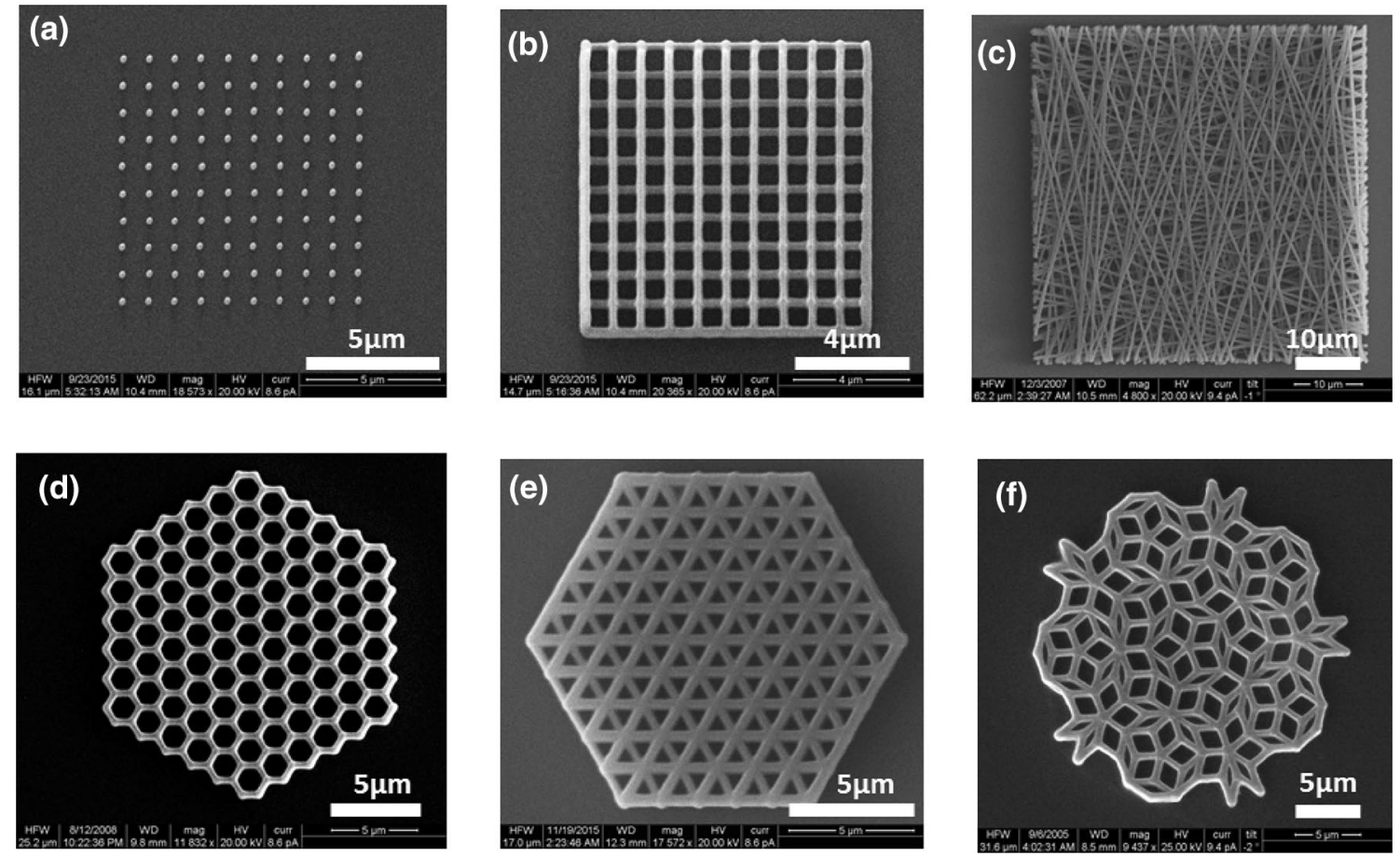

Fig. 1 SEM images of different 2D photonic structures fabricated by the two-photon polymerization method. a Direct square structure $(10 \times 10)$ voxels, $a_{1}=a_{2}=1 \mu \mathrm{m}$, b fishnet, $\mathbf{c}$ disordered woodpile, $\mathbf{d}$ honeycomb, e triangular, and $\mathbf{f}$ Penrose tiling photonic structures

SN Applied Sciences 
$\alpha_{\max }=p \frac{\pi}{4}, 0 \leq p \leq 1$. All structures have external size of $50 \times 50 \mu \mathrm{m}$ in the $x y$-plane.

We fabricate dielectric photonic structures as 2D arrays of scatterers with the square $C_{4 v^{\prime}}$ orthogonal $C_{2 v^{\prime}}$, hexagonal $C_{6 v}$ lattice symmetry, and the Penrose tiling pentagonal local $C_{5 v}$ symmetry. The number of scatterers varied from 25 to 10,000. The lattice parameters varied in different samples in the range of $0.5 \mu \mathrm{m} \leq a \leq 8.0 \mu \mathrm{m}$. The correspondence of the resulting materials to the designed structures was confirmed by scanning electron microscopy (SEM) technique.

\subsection{Optical setup}

A photograph of the experimental setup for optical diffraction measurements is shown in Fig. 2a. A glass substrate $\sim 1 \mathrm{~cm}^{2}$ in area with a set of microsamples was mounted in a precision holder used in X-ray diffraction studies. The samples were illuminated by a Nd laser with wavelength of $\lambda=0.53 \mu \mathrm{m}$. The laser beam was focused by a lens ( $25 \mathrm{~cm}$ focal length) onto microsamples at normal incidence. The optical system provided a full exposure of the sample (the laser spot at the samples surface was about $150 \mu \mathrm{m}$ in diameter), so that all the particles scattered light with the same intensity. The diffraction patterns were examined visually and photographed on a flat semitransparent screen placed behind the sample in a far-field region. The distance from the sample to the screen was about $20 \mathrm{~cm}$.

\section{Laue diffraction from 2D photonic structures}

\subsection{Laue diffraction: theoretical background}

For the analysis of Laue diffraction patterns from low-contrast 2D photonic structures, we use the Born approximation. In Born approximation, the diffraction intensity is determined by a product of the squares of the structure factor $S(q)$, the scattering form factor $F(q)$, and a polarization factor [9]. In our low-contrast case, it is sufficient to consider only the structure factor $S(q)$. For the 2D square structure, the position of each scatterer is determined by the $2 \mathrm{D}$ vector $\mathbf{r}_{i}=\mathbf{a}_{1} n_{1}+\mathbf{a}_{2} n_{2}, 0 \leq n_{j} \leq N_{j}$ and $N_{j}$ are integer. The diffraction angles and peak intensities become simple functions of the crystallographic 2D structure [10]:

$$
\begin{aligned}
S(\mathbf{q})= & \frac{\sin \left(N_{1} \mathbf{q} \mathbf{a}_{1} / 2\right)}{\sin \left(\mathbf{q} \mathbf{a}_{1} / 2\right)} \frac{\sin \left(N_{2} \mathbf{q} \mathbf{a}_{2} / 2\right)}{\sin \left(\mathbf{q} \mathbf{a}_{2} / 2\right)} \\
& \exp \left(i \frac{\left(N_{1}-1\right) \mathbf{q} \mathbf{a}_{1}+\left(N_{2}-1\right) \mathbf{q} \mathbf{a}_{2}}{2}\right) .
\end{aligned}
$$

Here, $\mathbf{q} \equiv \mathbf{k}_{i}-\mathbf{k}_{s}$ is the scattering vector, whereas $\mathbf{k}_{i}$ and $\mathbf{k}_{s}$ are the wave vectors of the incident and scattered waves.

To analyze the diffraction patterns, we consider the scattering from one-dimensional linear chain of scatterers first. The condition for the appearance of the Laue diffraction maxima for the linear chain and normal incidence is described by the simple formula: (a)

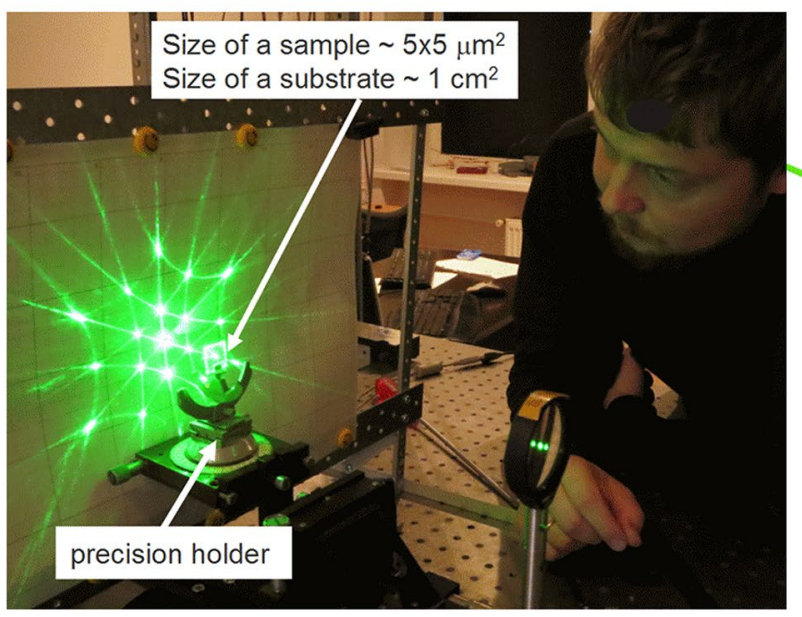

(b)

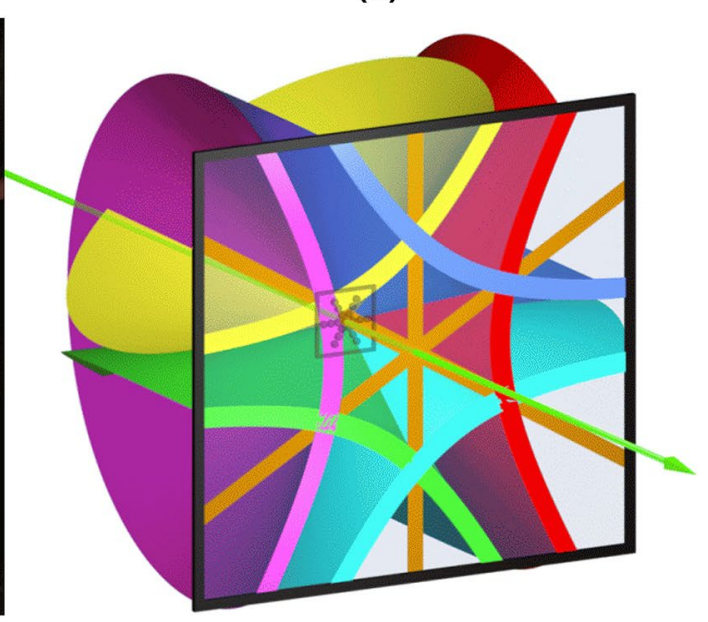

Fig. 2 a Experimental optical setup and pattern for zero-, firstand second-order Laue diffraction of monochromatic light $(\lambda=0.53 \mu \mathrm{m})$ from a triangular 2D structure were observed on a flat screen positioned behind the sample. b Schematic of the zero- and first-order Laue diffraction from the triangular structure is presented in Fig. 1e. Diffraction patterns (arcs and strips) on a flat screen are shown by thick lines. Scattered light is shown by different colors for clarity 
$\theta_{\mathrm{s}}=\cos ^{-1}(n \lambda / a)$

where $a=\left|\mathbf{a}_{1}\right|, \lambda$ is the wavelength of incident light, $\theta_{s}$ is the angle of scattering on the chain between vectors $\mathbf{a}_{1}$ and the wave vector of the scattered waves $\mathbf{k}_{s}$. Equation (2) defines the diffraction selection rules in relation to the ratio between $\lambda$ and $a$ because the inverse cosine function is defined in the interval from -1 to 1 only. For zero-order diffraction $(n=0)$, the angle of light scattering becomes $\theta_{s}=90^{\circ}$ and the scattering is observed for any ratio between $\lambda$ and $a$ in the plane perpendicular to the axis $\mathbf{a}$. Next, a pair of diffraction cones of the $n$-th order appears at $a>n \lambda$. For $\lambda<\alpha<2 \lambda$, we can distinguish in the diffraction patterns additionally to the plane $(n=0)$ a pair of cones $(n=1)$ that has the axes of symmetry coinciding with $\mathbf{a}$ and the apex angle of scattering $\theta_{s 1}=\cos ^{-1}(\lambda / a)$ (Fig. 2b). For $2 \lambda<\alpha<3 \lambda$, we can observe the plane and two pairs of cones with $\theta_{s 1}=\cos ^{-1}(\lambda / a)(n=1)$ and $\theta_{s 2}=\cos ^{-1}(2 \lambda / a)(n=2)$ (Fig. 2a) and so on.

We observed experimentally strong optical diffraction from rather small $(50 \times 50 \mu \mathrm{m})$ low-contrast dielectric 2D samples on a flat screen placed behind the sample (Fig. 2a). The ordered fishnet structure (Fig. 1b) can be considered as a structure composed of two sets of mutually orthogonal chains of scatterers along the $x$ - and $y$-axes.

For all ordered fishnets with the square symmetry $C_{4 v}$, the experimentally measured diffraction patterns demonstrate the same $C_{4 v}$ symmetry (Fig. 3). In Fig. 3a, for all samples, we can distinguish on the screen two types of the diffraction features: Two orthogonal strips that correspond to the zero-order scattering $(n=0)$ and two pairs of arcs that correspond to the first-order of scattering $(n= \pm 1)$. The patterns are formed by intersections of planes or cones with flat screen, as shown schematically in Fig. $2 \mathrm{~b}$. For the chain of scatterers with the lattice constant of $a_{1}=1 \mu \mathrm{m}$ and $\lambda=0.53 \mu \mathrm{m}$, the firstorder cones have angle of scattering $\theta_{s 1}=58^{\circ}$.

\subsection{Transformation from Laue diffraction to speckle patterns}

Figure 3 presents the results of experimental studies of light scattering from ordered and disordered woodpile thin slabs depending on the disorder parameter $p$, together with the SEM images of the corresponding structures. When the random fluctuations of the orientation for both $x$-oriented and $y$-oriented individual rods are introduced, the diffraction patterns changed dramatically: Stripes and arcs become randomized and a granular distribution of light intensity appears through the entire screen (Fig. 3b-f). For disordered structure, light from different points of the sample traverses different optical path lengths to reach the screen. As a result, a laser speckle appears on the screen which is a random interference effect that gives a high-contrast granular distribution (a)

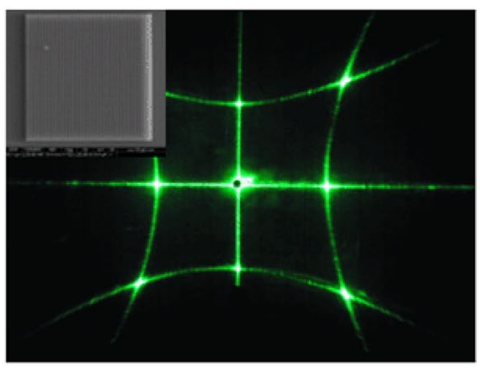

(d)

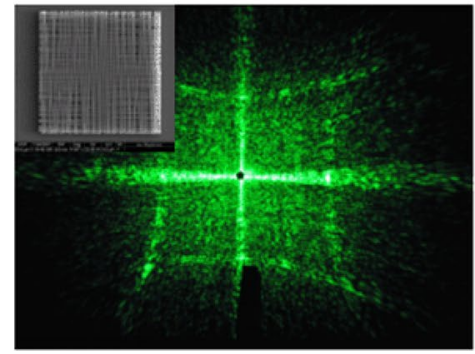

(b)

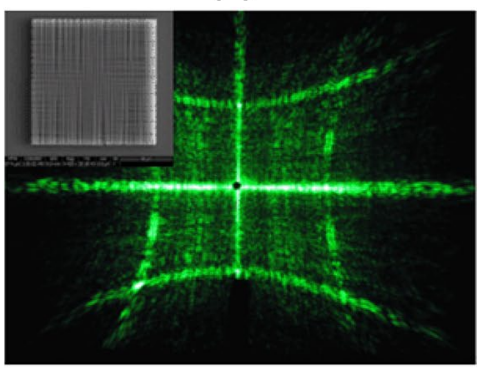

(e)

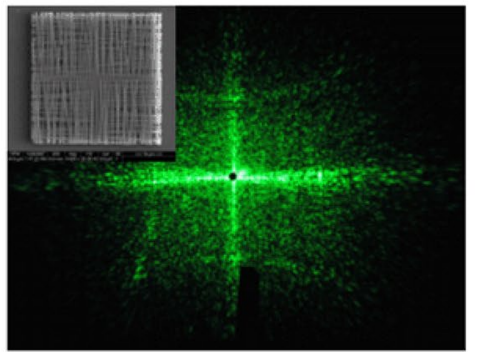

(c)

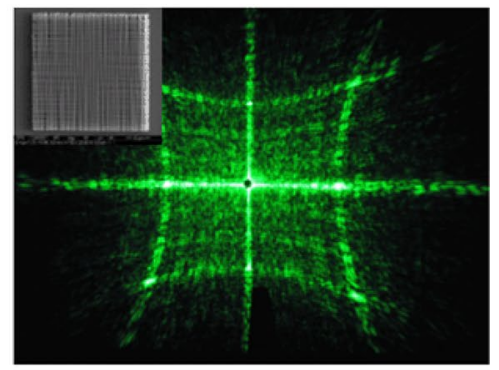

(f)

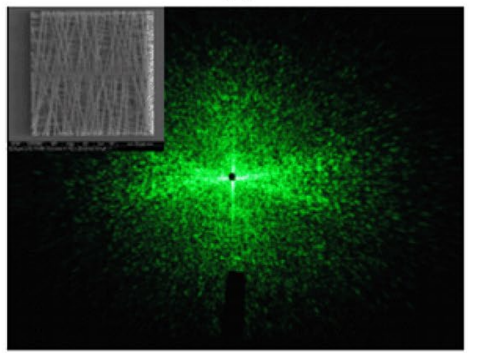

Fig. 3 Diffraction pattern evolution from ordered $\mathbf{a}$ to disordered b-f woodpile thin slabs with normal distribution of the disordered parameter $p$. a $p=0$, b $p=0.02$, c $p=0.04$, d $p=0.06$, e $p=0.1$, f $p=0.5$. The lattice parameters of the ordered sample $a_{x}=a_{y}=1 \mu \mathrm{m}$, the number of layers along the $z$-axis $N=8$ for all samples. The patterns are observed on a flat screen positioned behind the sample. $\lambda=0.53 \mu \mathrm{m}$

\section{SN Applied Sciences}


of scattering intensity. The speckle diffraction pattern is one of the most remarkable properties of photonic glasses [11], the disordered woodpile we be classified as a new type of such photonic structures.

Figure 4 presents results of the diffraction study on anisotropic glassy woodpiles. The anisotropic structure is designed with disorder in rods which initially oriented along $x$-axis while all rods oriented along $y$-axis are ordered. From Fig. 4e, it is clearly seen that the arcs from ordered set of rods (a rod marked with the red rectangle in Fig. 4d) becomes randomized and hardly observed while the cones from disordered set of rods (a rod marked with the yellow rectangle in
Fig. 4d) remained strong and bright. To explain this result, we have carried out the calculations of the diffraction patterns using two different models of scatterers. For the first model, we consider each dielectric rod as a set of 1000 coaxial equidistant point scatterers ("rod model"). Such model (Fig. 4i) fails to describe the experimental patterns in Fig. 4e. For the second model, we assume that only points of rods intersections define the diffraction patterns ("points of intersections model"). The results of calculations using the "points of intersections" model are in excellent agreement with experimental data. The reason is that all points of intersections owned by each $x$-oriented rod are distributed equidistantly (blue (a)

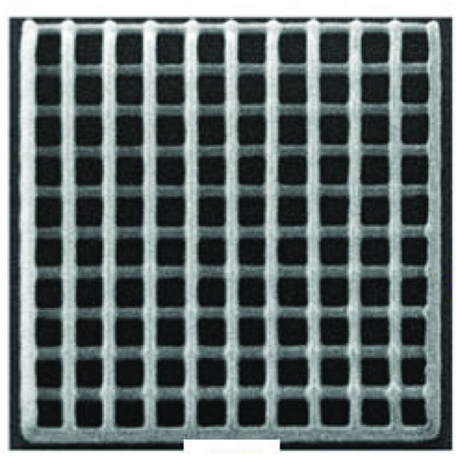

(b)

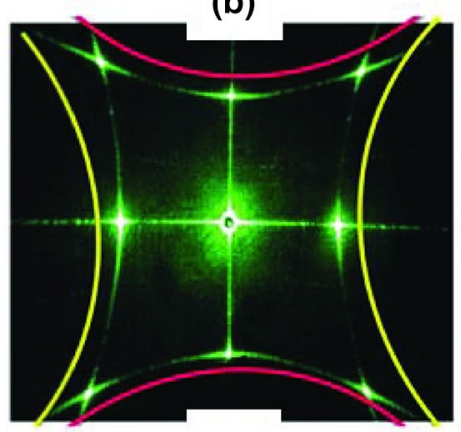

(c)

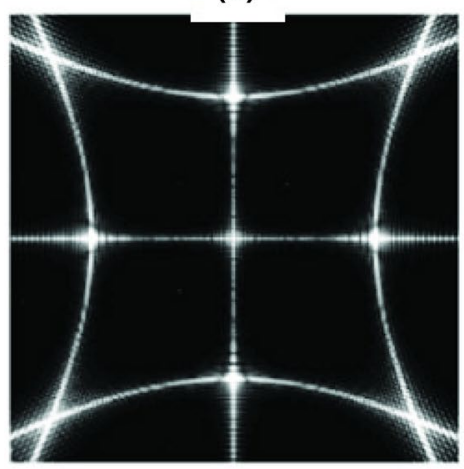

(d)

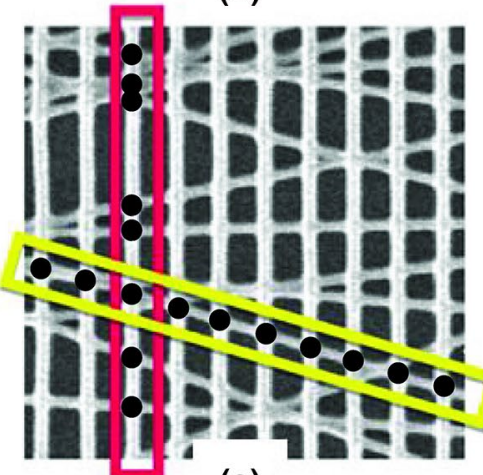

(e)

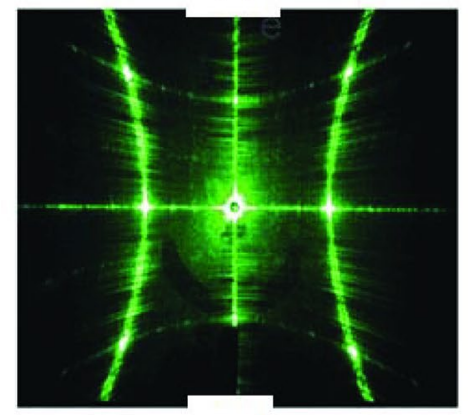

(f)
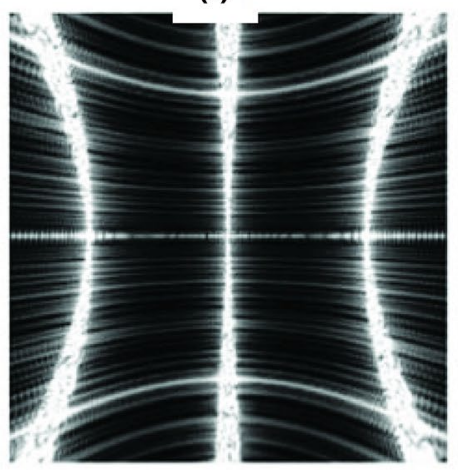

(g)

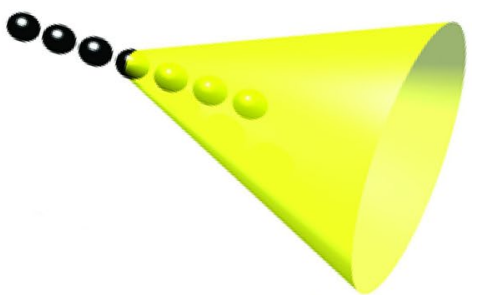

(h)

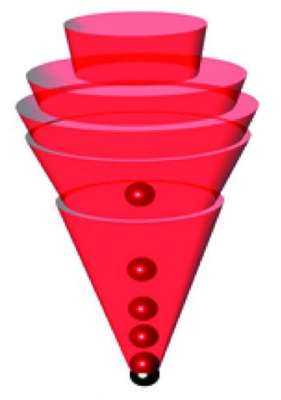

(i)

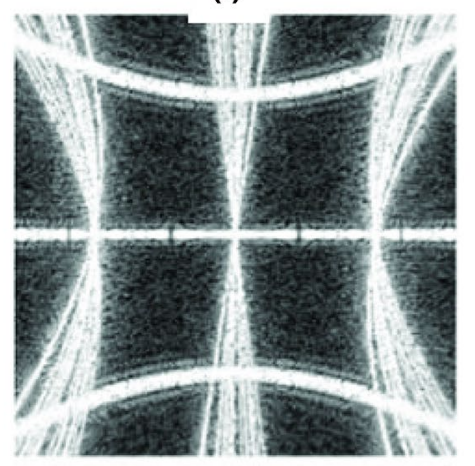

Fig. 4 SEM images of the square ordered $\mathbf{a}$ and $x$-disordered $\mathbf{d}$ woodpile-type photonic structures. b, e Experimentally measured diffraction patterns corresponding to the upper structures. c, $\mathbf{f}$ Diffraction patterns numerically calculated using the "points of intersections model". g, h Schematic of the zero-order $(n=0)$ and first-order $(n= \pm 1)$ Laue diffraction from the horizontally $(\mathbf{g})$ and vertically (h) oriented chains of scatterers. Yellow color corresponds to scattering from horizontal (disordered) rods, and red color corresponds to scattering from vertical (ordered) rods. i Diffraction patterns numerically calculated using the "rod model" 

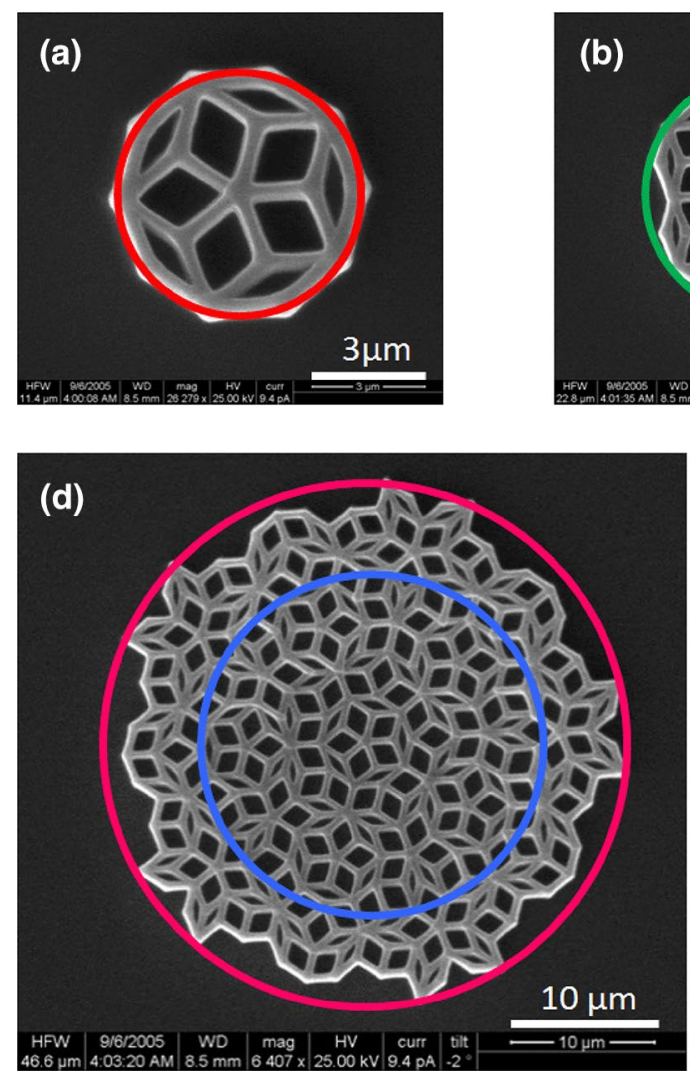
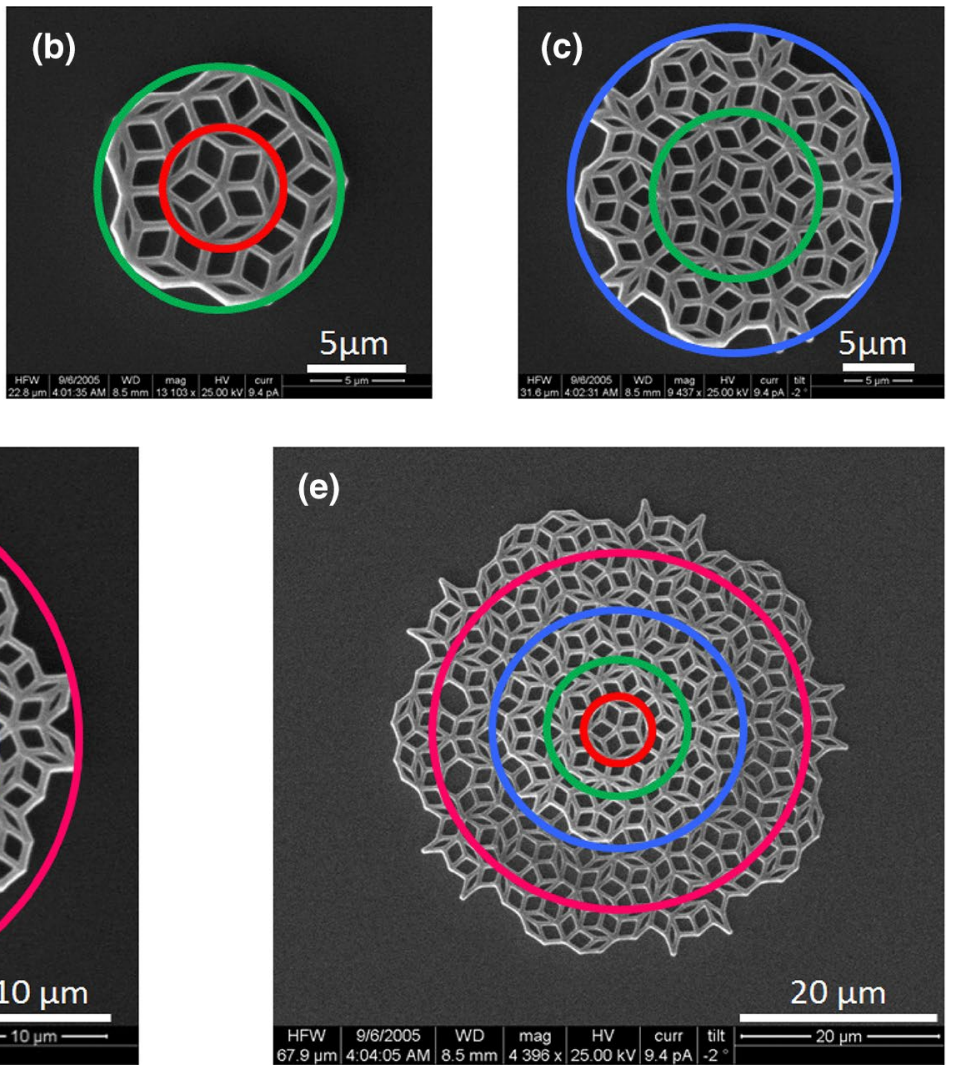

Fig. 5 SEM images of Penrose tiling photonic structures. Colored circles show substitution rules for constructing a 2D Penrose quasicrystal

Fig. 6 Photographs of two Penrose tiling photonic structures $\mathbf{a}, \mathbf{b}$ and corresponding experimentally measured Laue diffraction patterns $\mathbf{c}, \mathbf{d}$ $\lambda=0.53 \mu \mathrm{m}$
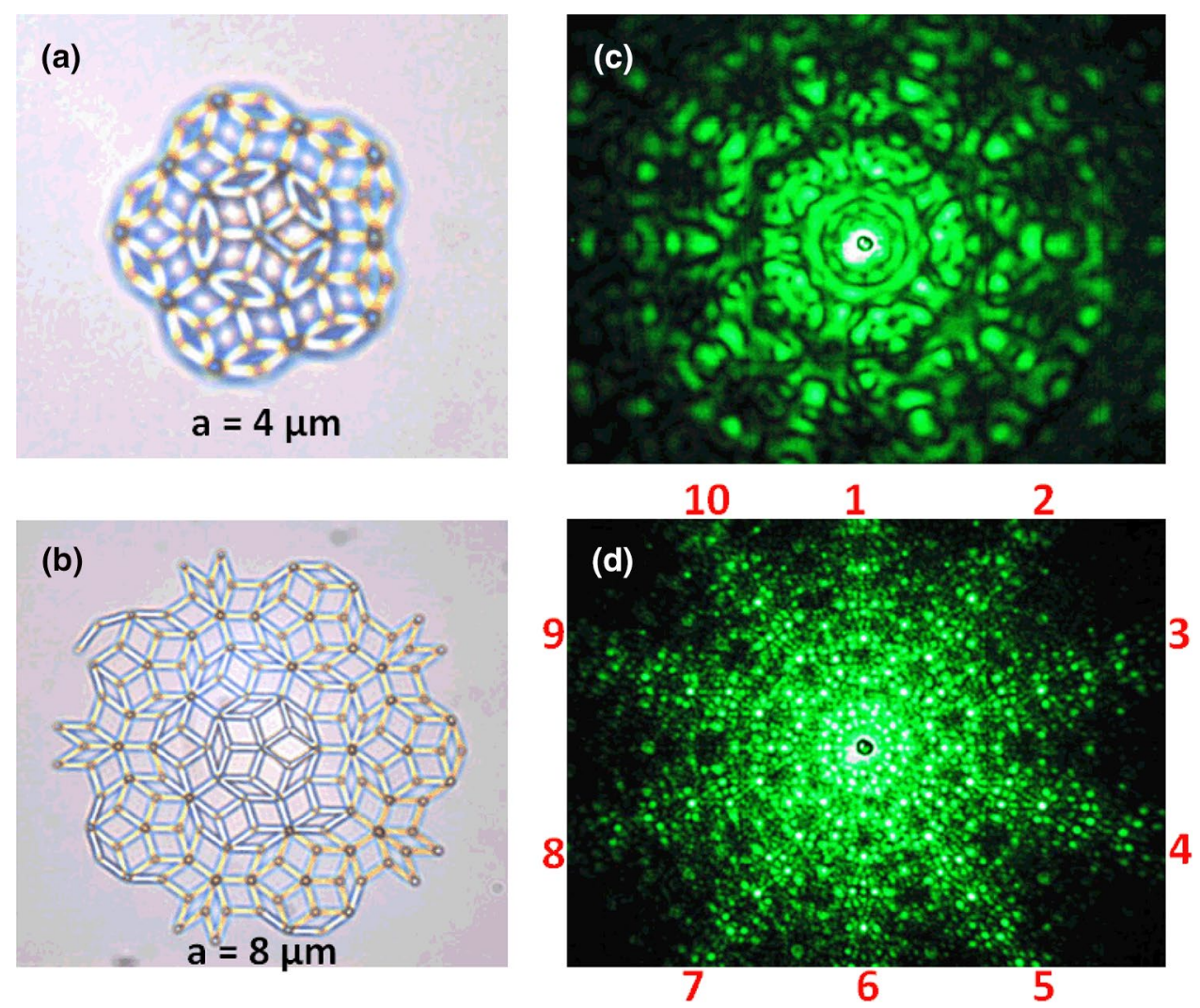

SN Applied Sciences 
points in Fig. 4c) due to equidistant arrangement of the $y$-oriented ordered set of rods. Therefore, each $x$-oriented rod gives rise to perfect (yellow) arcs. In contrast, because of the disorder in the $x$-oriented set of rods, all interscatterer distances in $y$-direction become randomized resulting in the destruction of the perfect (red) arcs.

\subsection{The Penrose tiling}

The Penrose tilings were described for the first time in a paper with a title "The role of aesthetics in pure and applied mathematical research" [12] that emphasizes the extraordinary structure of these $2 \mathrm{D}$ quasicrystalline lattices $[13,14]$. We note that the concept of quasicrystal as a nonperiodic structure with perfect long-ranged order was brought in solid-state physics in 1984 [15], 10 years after Roger Penrose's publication [12]. The Penrose tiling can be thought of as a generalization of the Fibonacci lattice to the $2 \mathrm{D}$ case [16].

We have fabricated a set of the Penrose photonic quasicrystals using the direct laser writing technique. A Penrose quasicrystal structure can be built according to the additive rules as shown in Fig. 5. Two types of rhombuses having sides of the same length but different angles at the vertices constitute a Penrose tiling. Large rhombuses with $a_{\mathrm{big} 1}=72^{\circ}$ and $a_{\text {big } 2}=108^{\circ}$ angles form a central star, and small rhombuses with $a_{\mathrm{sm} 1}=36^{\circ}$ and $a_{\mathrm{sm} 2}=144^{\circ}$ angles form the next layer of the structure (Fig. 5a), and so on (Fig. 5b-d).

We also demonstrate two images of Penrose tilings taken with a conventional optical photograph, Fig. 6a, b. Figure $6 c$, d demonstrate experimentally measured Laue diffraction patterns. As theoretically expected, a fivefold orientational symmetry of the Penrose tiling gives rise to a tenfold orientational symmetry of the diffraction images as demonstrated by the numbering in Fig. $6 \mathrm{~d}$.

\section{Conclusions}

We demonstrate that optical diffraction experiments offer a variety of information on the mechanisms of light scattering from 2D ordered and disordered dielectric photonic structures. The experiments were carried out on different 2D photonic structures fabricated using direct laser writing technique, and the results were discussed on the basis of the Laue equations in the Born approximation. We visualized the diffraction patterns for 2D photonic structures on a flat screen placed behind the sample. We have demonstrated the mechanism for the formation of optical diffraction patterns from disordered woodpile-type photonic structures. Our calculated results are in good agreement with experiment. We also found the tenfold orientational symmetry of the diffraction patterns of Penrose tilings.
Funding Authors acknowledge support by Goszadanie (Grant Nos. 0040-2019-0006). M.V.R. and A.D.S acknowledge support by the Ministry of Education and Science of the Russian Federation (3.1500.2017/4.6)

\section{Compliance with ethical standards}

Conflict of interest The authors declare that they have no conflict of interest.

\section{References}

1. Farsari M, Chichkov BN (2009) Two-photon fabrication. Nat Photon 3:450-452

2. Fischer J, Wegener M (2013) Three-dimensional optical laser lithography beyond the diffraction limit. Laser Photon Rev 7:22-44

3. Sekkat Z, Kawata S (2014) Laser nanofabrication in photoresists and azopolymers. Laser Photon Rev 8:1-26

4. Rybin MV, Samusev KB, Lukashenko SYu, Kivshar YS, Limonov MF (2016) Transition from two-dimensional photonic crystals to dielectric metasurfaces in the optical diffraction with a fine structure. Sci Rep 6:30773

5. Sinelnik AD, Rybin MV, Lukashenko SY, Limonov MF, Samusev KB (2017) Optical properties of honeycomb photonic structures. Phys Rev A 95:063837

6. Rybin MV, Sinev IS, Samusev AK, Samusev KB, Trofimova EY, Kurdyukov DA, Golubev VG, Limonov MF (2013) Dimensionality effects on the optical diffraction from opal-based photonic structures. Phys Rev B 87(12):125131

7. Briers JD (2001) Laser Doppler, speckle and related techniques for blood perfusion mapping and imaging. Physiol Meas 22:R35-R66

8. Brüser B, Staude I, von Freymann G, Wegener M, Pietsch U (2012) Visible light Laue diffraction from woodpile photonic crystals. Appl Opt 51:6732-6737

9. Ziman JM (1979) Models of disorder: the theoretical physics of homogeneously disordered systems. Cambridge University Press, Cambridge

10. Guinier A (1994) X-ray diffraction in crystals, imperfect crystals, and amorphous bodies. Courier Corporation

11. Garcia PD, Sapienza R, Blanco A, López C (2007) Photonic glass: a novel random material for light. Adv Mater 19:2597

12. Penrose $R$ (1974) The role of aesthetics in pure and applied mathematical research. Bull Inst Math Appl 10:266

13. Shir D, Liao H, Jeon S, Xiao D, Johnson HT, Bogart GR, Bogart KHA, Rogers JA (2008) Three-dimensional nanostructures formed by single step, two-photon exposures through elastomeric Penrose quasicrystal phase masks. Nano Lett 8:2236

14. Kaliteevski MA, Brand S, Abram RA, Krauss TF, Millar P, De La Rue RM (2001) Diffraction and transmission of light in lowrefractive index Penrose-tiled photonic quasicrystals. J Phys Condens Matter 13:10459

15. Levine D, Steinhardt PJ (1984) Quasicrystals: a new class of ordered structures. Phys Rev Lett 53:2477

16. Poddubny AN, Ivchenko EL (2012) Quasicrystalline photonic structures: between order and disorder. In: Limonov MF, Rue RDL (eds) Optical properties of photonic structures: interplay of order and disorder. CRC Press, New York

Publisher's Note Springer Nature remains neutral with regard to jurisdictional claims in published maps and institutional affiliations. 